\title{
Examen especial a la cuenta de depósitos a plazo fijo de BanEcuador sucursal Cuenca, por el período comprendido entre el 01 de enero al 31 de diciembre de 2018
}

Special examination of BanEcuador branch office Cuenca deposit account, from January 1st to December 31st, 2018.

Rosa Liliana Yuquilima Heras. ${ }^{1}$, Cecilia Ivonne Narváez Zurita. ${ }^{2}$, Mireya Magdalena Torres Palacios. ${ }^{3}$ \&Yanice Licenia Ordoñez Parra. ${ }^{4}$

DOI: $\underline{\text { https://doi.org/10.33262/visionariodigital.v3i2.1.587 }}$

\begin{abstract}
.
In this article we will present the findings on the special examination to BANECUADOR fixed-term deposits account. This investigation was carried out in Cuenca city, from January 1st to December 31st, 2018. The examination was carried out in accordance with the Internal Control Standards for entities, public sector bodies and private law individuals that make use of public resources, according to agreement 039-CG-2009, issued in the Official Register No. 78, Ecuadorian Government Auditing Standards (NEAG) issued by Official Registry Supplement 6 of October 6, 2002 and Government Financial Audit Manual (MAFG) Agreement 016-CG-2001 published in the Official Register No. 407, where control tests were carried out and substantive tests, which allowed to determine the effectiveness and efficiency of the operations, reliability of the information and compliance with applicable laws and regulations, in order to issue recommendations to the Financial-Accounting Management and the sub processes related to fixed term deposits.
\end{abstract}

\footnotetext{
${ }^{1}$ Universidad Católica de Cuenca, Posgradista Maestría en Contabilidad y Auditoría, Cuenca, Ecuador, rlyuquilimah085@psg.ucacue.edu.ec

${ }^{2}$ Universidad Católica de Cuenca, Subdirección de Posgrado, Cuenca, Ecuador, inarvaez@ucacue.edu.ec

${ }^{3}$ Universidad Católica de Cuenca, Subdirección de Posgrado, Cuenca, Ecuador, mireya.torres@ucacue.edu.ec

${ }^{4}$ Universidad Católica de Cuenca, Subdirección de Posgrado, Cuenca, Ecuador, jordonezp@ucacue.edu.ec
} 
Keywords: special examination, substantive tests, control tests, fixed term deposits, internal control.

\section{Resumen.}

En el presente artículo se dará a conocer los resultados del examen especial realizado a la cuenta de depósitos a plazo fijo de BANECUADOR, investigación que se llevó a cabo en la ciudad de Cuenca, en el período del 01 de enero al 31 de diciembre de 2018. El examen se efectuó de acuerdo a las Normas de Control Interno para las entidades, organismos del sector público y personas de derecho privado que dispongan de recursos públicos, según acuerdo 039-CG-2009, emitido en el Registro Oficial $N^{\circ} 78$, Normas Ecuatorianas de Auditoría Gubernamental (NEAG) emitido en el Registro Oficial Suplemento 6 de 6 de octubre de 2002 y Manual de Auditoría Financiera Gubernamental (MAFG) Acuerdo 016CG-2001 publicado en el Registro Oficial $N^{\circ} 407$, donde se realizó pruebas de control y pruebas sustantivas, que permitieron determinar la eficacia y eficiencia de las operaciones, fiabilidad de la información y cumplimiento de leyes y normas aplicables, con el objeto de emitir recomendaciones a la Dirección Financiera-Contable y a los subprocesos relacionados con los depósitos a plazo fijo.

Palabras Claves: Examen especial, pruebas sustantivas, pruebas de control, depósitos a plazo fijo, control interno

\section{Introducción}

Con la finalidad de lograr una mayor comprensión referente a los fundamentos teóricos del examen especial a la cuenta de depósitos a plazo fijo, es fundamental analizar las conceptualizaciones que se involucran en el estudio.

El examen especial realizado por la Contraloría, como parte de la auditoría gubernamental, es el que verificará, estudiará y evaluará aspectos limitados o de una parte de las actividades relativas a la gestión financiera, administrativa, operativa y medio ambiental; con posterioridad a su ejecución, aplicará las técnicas y procedimientos de auditoría, de la ingeniería o afines, o de las disciplinas específicas, de acuerdo con la materia de examen y formulará el correspondiente informe que deberá contener comentarios, conclusiones y recomendaciones (Contraloría General del Estado , 2003).

Por lo expuesto, la realización del examen especial a la cuenta de depósitos a plazo fijo en una institución financiera permitirá evaluar el cumplimiento de las normativas y procedimientos, un mejor manejo de la misma y una información confiable y oportuna, que ayudará, a su vez, a emitir un Informe que contenga las conclusiones y recomendaciones para la organización. 
Montilla Galvis y Herrera Marchena (2006) consideran a la auditoría como una actividad antigua y nace en el conocimiento y la práctica de la contabilidad, respecto a la representación macroeconómica. La auditoría reconoce, históricamente, a la denominada auditoría pública y gubernamental, que se encargaba del control de las haciendas públicas y todas las cuentas de los gobiernos de esa época; mientras tanto, en el campo microeconómico, se establece la auditoría privada y esta se encarga de la localización de fraudes, errores y todo desfase en las cuentas de las distintas organizaciones privadas para poder emitir una opinión sobre los estados financieros.

Acorde al progreso de la sociedad y los avances, que cada vez eran mayores, los gobiernos comienzan a ir sistematizando los actos del comercio y desarrollando las teorías impositivas; se enfocaban también en cada una de las prácticas comerciales y tributarias, en la contabilidad, lo cual les imponía un rol social generador de toda la información económica confiable, que la utilizaban los comerciantes para realizar sus actos de comercio y, además, era utilizada en los gobiernos para el cálculo del valor de los gravámenes para tener un valor más exacto y razonable para el cobro de los mismos (Sandoval, 2012).

La auditoría también ha ido registrando avances, con la finalidad de poder realizar revisiones y comparaciones tanto en la contabilidad como en sus componentes y derivaciones, y, de este modo, se fueron también instituyendo las normalizaciones, en este caso, de los gobernantes y agremiaciones que practicaban tales oficios.

En este contexto los auditores, independientemente, se fueron especializando sobre la importancia de cómo se iba desarrollando un buen sistema de control interno y su correspondencia con el alcance de todas las pruebas que se efectúan en la práctica de la auditoría independiente; las administraciones se mostraron afiliadas del crecimiento de departamentos de auditoría dentro de las organizaciones de los clientes, $y$, estos departamentos serían los que se encargarían del desarrollo y mantenimiento de cada uno de los procesos del control interno.

Mantilla, Herrera, y Pinilla (2005) hacen hincapié en que la auditoría se erige como un campo de estudio multidisciplinario, pues las haciendas veían una necesidad de verificación de fraudes, en donde primaba la capacidad de los conocimientos de cada uno de los objetivos sobre los diferentes objetos de estudio; mientras tanto, según se fueron desarrollando todos los avances, empezó a nacer la necesidad de que un contador público pueda desempeñar el trabajo de auditor en las organizaciones para poder detectar cualquier falla en cada proceso presentado y poder emitir un informe para la toma de decisiones.

Para Santillana (2004) la auditoría significa verificar que la información financiera, administrativa y operacional de una entidad sea confiable, veraz y oportuna; en otras palabras, es revisar que los hechos, fenómenos y operaciones se den en la forma en que fueron planeados, que las políticas y lineamientos se hayan observado y respetado, que se cumpla con las obligaciones fiscales, jurídicas 
y reglamentarias en general. El objetivo de la auditoría consiste en apoyar a los miembros de la empresa en el desempeño de sus actividades. Para ello, les proporciona análisis, evaluaciones, recomendaciones, asesoría e información concerniente a las actividades revisadas (Cuéllar, 2003).

En general, cuando de los entes económicos u organizaciones se trata, la auditoría apoya desde el nivel operativo, pasando por las diferentes instancias de la empresa, entre ellas, la administración, hasta llegar a la alta gerencia, nivel donde el auditor se debe vincular con el ente, para tener una posición de independencia o staff; de este modo, la misma se constituye como una herramienta de control y supervisión, que contribuye a la creación de una cultura de disciplina en la organización, permitiendo descubrir las desviaciones en las estructuras y las vulnerabilidades existentes en el ente.

La auditoría, más que un sistema de cumplimiento para una organización, debe ser considerado un sistema de control de calidad para cada uno de los departamentos o cuentas a auditar, lo que permitirá evaluar el sistema de calidad en las áreas auditadas para poder visualizar el cumplimiento de logros de los mismos. La auditoría en sí, se constituye en una herramienta de control y supervisión que contribuye a la creación de una cultura de disciplina en a organización, y permite descubrir fallas en las estructuras o vulnerabilidades existentes en la entidad, a la vez que se aplica dentro de un período determinado para conocer sus restricciones, problemas y deficiencias, como parte de evaluación en la organización, para luego emitir un informe con conclusiones y recomendaciones para la toma de decisiones (Mejía, Montilla, y Montes, 2005).

En la actualidad, su metodología y procedimientos han venido adaptándose a la medida del crecimiento y la complejidad de las áreas o actividades auditadas, por ello, y con especial reconocimiento, es muy importante tener en cuenta que los resultados esperados, se obtienen de la metodología que se utilice para la ejecución de una auditoría, la cual debe estructurarse con la normatividad vigente para su aplicación, emitiendo, de una forma objetiva, un dictamen veraz, que permita a la organización hacer mínimas las brechas existentes entre el riesgo y el control, permitiendo, así, una mayor probabilidad en el cumplimiento de los objetivos y, por ende, una mayor posibilidad de éxito empresarial.

La auditoría se clasifica de diferentes formas y depende de lo que se desea indagar.

Auditoría externa: se realiza un examen a las cuentas de una empresa, mediante pruebas selectivas, que ayudará a presentar los rubros revisados; este es realizado por un auditor fuera de la empresa, que no mantiene vinculación alguna con la misma, que presta sus servicios independientemente, basados en la auditoría y el atestiguamiento.

Auditoría interna: es ejecutada por un empleado dependiente de la misma empresa, para poder garantizar que cada una de las operaciones se estén llevando de una manera eficiente y eficaz, 
proponiendo soluciones a los problemas. La auditoría interna comprende también los siguientes tipos de auditoría: administrativa, operacional y financiera (Cañibano, 1996).

Auditoría financiera: el auditor revisa y verifica la aplicación correcta de cada uno de los registros contables y todas aquellas operaciones financieras de la organización (Defliese, Jaenicke, Gnospelius, y Sullivan, 1996).

Auditoría administrativa: el auditor realiza una revisión muy sistemática y exhaustiva a las actividades administrativas de la organización a cada uno de los integrantes y el cumplimiento de cada una de las actividades administrativas que sistematizan las operaciones (Ibídem)

Auditoría operacional: se realiza una revisión muy sistemática y determinada a cada una de las actividades de la empresa, con el fin de poder verificar y evaluar el desarrollo de cada una de las operaciones.

Auditoría gubernamental: el auditor realiza una revisión de cada una de las actividades en una entidad gubernamental; cabe indicar que, en este tipo de auditoría, se puede realizar un examen especial para un mejor análisis, para lo cual se deberá aplicar técnicas y procedimientos a fin de poder realizar el informe, (Cañibano, 1996).

Por lo expuesto, puede ocasionarse una confusión en la auditoría, razón por la que, en esta investigación, se analizan las más relevantes y utilizadas, con lo cual se puede deducir que, en la auditoría financiera, operacional y gubernamental, realizar un examen especial, permite un análisis más profundo, para así poder informar sobre todos los hallazgos y las recomendaciones de los problemas encontrados en la organización. Se puede afirmar que el examen especial permite profundizar, de una manera más clara, el cumplimiento de manuales y procedimientos o manejo de las cuentas.

Como parte de la auditoría gubernamental el examen especial permite evaluar aspectos limitados de un departamento o cuenta específica, con la finalidad de dar atención en las falencias encontradas y las causas respectivas; a su vez, estas falencias, mediante el examen especial, se las podrá corregir para poder mantener la organización de una manera más eficiente y eficaz.

Mediante el examen especial, como parte de la auditoría gubernamental, se evalúa, verifica y estudia aspectos concretos o una parte de las actividades concernientes a la gestión administrativa, financiera y operativa. Posterior a su implementación, debe aplicar los procedimientos y las técnicas de auditoría, ingeniería o afines, o de las disciplinas concretas, acordes con la materia del examen y debe estructurar también el informe pertinente, el mismo que debe contener observaciones, conclusiones y recomendaciones (Moore Stephens, 2019). El examen especial, como parte de la auditoría gubernamental verificará, estudiará y evaluará aspectos limitados o de 
una parte de las actividades relativas a la gestión financiera, administrativa, operativa y medio ambiental, con posterioridad a su ejecución (Contraloría General del Estado 2003).

La realización de los exámenes especiales en ecuador se basan en el plan anual de control de la Contraloría y los resultados de una verificación preliminar por pedidos o denuncias los auditores efectuarán el trabajo del examen especial tomando en cuenta el departamento al que va ser realizado. El examen especial debe tener objetivos, sistemáticos y profesionales, y, es muy importante, conocer todos los procesos y técnicas a aplicar, así como su alcance y naturaleza.

El examen especial se realiza con el objetivo principal de conocer la entidad y prestar atención minuciosa a todas las políticas y procedimientos de la misma; otros objetivos que pueden mencionarse son: observar cómo se desarrollan las actividades; revisar archivos corrientes y permanentes.

Para realizar el examen especial, se requiere recopilar la siguiente información y documentación:

a) Ubicación de la organización

b) Visión, misión y objetivos de la organización.

c) Estructura orgánica.

d) Funciones de la estructura orgánica.

e) Filosofía de la organización.

f) Políticas y reglamentos de la organización.

g) Ilustración de la actividad principal y operaciones importantes de la organización.

h) Situación financiera actual; organización y estructura del personal; y demás documentos con que cuenta la organización (Moore Stephens, 2019).

Luego de esta revisión, es preciso conocer la estructura de control interno organizacional y su funcionamiento, identificando los componentes importantes para la evaluación de control interno y que, posteriormente, serán sometidos a pruebas y procedimientos de auditoría; por último, se define el objetivo y la estrategia general del examen especial.

Las personas que realizan estos exámenes, al igual que en las demás auditorías, deben ser multidisciplinarias, de acuerdo al tema a analizarse y la complejidad y profundidad de la revisión efectuada, dejando evidencia de cada procedimiento realizado y cumpliendo con las etapas de planificación, ejecución del trabajo, comunicación de resultados parciales y elaboración del informe, con sus respectivos comentarios, conclusiones y recomendaciones.

Los procedimientos para llevar a cabo un examen especial son similares a los de una auditoría. Los pasos más importantes son:

- Planificación preliminar 
- Planificación especifica

- Ejecución

- Informe

La planificación preliminar es la primera fase para la emisión formal de la orden de trabajo del equipo auditor y la notificación de inicio del examen a los servidores involucrados en el proceso de la auditoría posteriormente se realiza un programa de planificación preliminar, en donde se detallan las actividades a realizarse por los auditores en las fechas indicadas

La misma que se sustenta en la planificación anual de control de la entidad, en donde se consigue información utilizando: exploración de archivos, conocimiento de las instalaciones y entrevistas a los funcionarios responsables de las actividades, lo que permitirá, de modo general, las condiciones existentes, así como la consecución de apertura y disposición para ejecutar la labor de auditoría.

\section{Procedimientos específicos:}

- Solicitar información general, presupuestos y estados financieros.

- Solicitar información de contratos de depósitos a plazo fijo.

- Revisar el Reglamento Interno Organizacional y Funcional

- Redactar el reporte de planificación preliminar.

Luego de ejecutar las actividades en concordancia al programa de planificación preliminar, se emite un reporte, que conste de: antecedentes de la auditoría, motivo de la auditoría, objetivos de la auditoría, alcance, conocimiento de la institución, grado de confiabilidad de la información, monto de recursos analizados, servidores relacionados, con la respectiva matriz preliminar de riesgos.

Planificación especial, en esta fase se evalúa el control interno mediante la aplicación de las Normas de Control Interno emitidas por la Contraloría General del Estado, con la finalidad de identificar y calificar riesgos del examen y establecer el nivel de confianza de la auditoría. La ejecución de esta fase se efectúa de acuerdo a un programa de planificación específica.

\section{Programa de planificación, objetivos:}

- Evaluar el control interno de los depósitos a plazo fijo.

- Analizar la información financiera-presupuestaria de los depósitos a plazo fijo.

- Evaluar y calificar el riesgo del examen e identificar áreas críticas

- Establecer el enfoque de la auditoría 


\section{Procedimientos:}

- Realizar la evaluación del control interno a los subprocesos relacionados con los depósitos a plazo fijo

- Efectuar el análisis del estado de situación financiera, presupuesto, aplicando indicadores financieros presupuestarios y analizar sus resultados

- Elaborar un memorando de planificación específica

- Elaborar la matriz de planificación específica

- Elaborar el programa de la auditoría

- Redactar un informe de control interno

Ejecución, consiste en la aplicación de los procesos fijados en los programas de auditoría, en donde el auditor despliega los hallazgos de relevancia de las áreas o componentes críticos, en el que establece los atributos de condición, criterio, causa y efecto de los problemas o desviaciones identificadas; estos hallazgos son respaldados en papeles de trabajo, cuya evidencia de auditoría es pertinente, suficiente, adecuada y competente, que respalda la opinión y el informe (Contraloría General del Estado, 2003).

- Papeles de trabajo, comprende: cédulas, programas de trabajo de auditoría, índice, notas específicas y documentación selecta. Estos papeles avalan las pruebas realizadas, a partir de las cuales se derivan las conclusiones (Sotomayor, 2008).

- Organización de los papeles de trabajo del examen especial, pueden ser organizados del siguiente modo:

En archivo corriente: posee fundamental información sobre los resultados de la actividad auditora, que fundamentan la opinión de la información examinada; se pueden citar, entre otros: evidencia obtenida, procedimientos aplicados (pruebas de cumplimiento o sustantivas).

- Tipos de pruebas en el examen especial, las pruebas del examen especial permiten crear evidencia y generalmente se realizan en la fase de ejecución, pero, en ciertos casos, se efectúa en la planificación. Los tipos de prueba pueden ser:

Prueba de control: concede al auditor evidencia sobre la existencia de controles adecuados en la institución auditada. Esta prueba se divide en:

a) Pruebas de cumplimiento: permiten verificar el funcionamiento de los controles prescritos por la entidad;

b) Pruebas de observación: permiten verificar los controles en los procesos que no disponen de evidencia documentada. 
- Pruebas sustantivas: son las indagaciones, opiniones de los servidores, el proceso de diagnóstico, las observaciones, actualizaciones de los sistemas y formación, seguimiento de documentos referidos a transacciones, procedimientos analíticos (permite evaluar, de manera efectiva, la razonabilidad de los montos en los estados financieros), constataciones, entre otros. Esta prueba proporciona evidencia directa al auditor.

El auditor efectúa estos tipos de pruebas, luego de haber evaluado el control interno y obtener sus resultados (Manual de Auditoría Financiera Gubernamental, 2001).

- Técnicas del examen especial, La investigación del examen especial conlleva una metodología definida (planificación), que requiere del empleo de ciertas técnicas, entre las que se puede mencionar:

- Entrevista: entablar comunicaciones con el personal involucrado de manera directa, con el fin de obtener información.

- Observación directa: permite percibir los hechos que se suscitan en la entidad, relacionados con la evaluación y el ambiente en el que opera.

- Cuestionario: formulario diseñado, ya sea con anticipación o al momento de su utilización, para recabar información sin límites, tanto del tema a evaluar como de aspectos generales de la entidad.

- Investigación documental: examinar, indagar, descubrir y averiguar detalles de un tema concreto, mediante documentos pertenecientes a la institución, mismos que, al ser analizados, aportarán elementos de juicio en la evaluación que se ejecuta.

- Análisis administrativo: posee técnicas diversas, que se aplican de acuerdo al tema evaluado, al tiempo y la disposición de la entidad, entre las que se puede contar las de tipo administrativo y las de carácter operacional (Sotomayor, 2008).

- Los hallazgos del examen especial, hacen relación a posibles irregularidades o deficiencias registradas como resultado de la aplicación de procedimientos de auditoría. Los resultados de las tareas de control efectuadas, serán analizados sólo con las personas involucradas en los hechos examinados y con las autoridades de la entidad.

Informe, Esta es la última fase del proceso de auditoría y se efectúa también durante la ejecución del examen. En esta fase es fundamental la comunicación continua con los funcionarios vinculados al examen de la auditoría, pues les mantiene informados sobre las desviaciones y deficiencias detectadas, con el objetivo de que presenten justificativos u oportunas acciones correctivas. En esta fase se realiza la redacción y revisión final del borrador hecho en el proceso del examen; su objetivo es declarar concluido el trabajo de auditoría con previa convocatoria, donde se comunica los resultados con la lectura del informe, a las autoridades, servidores y ex servidores responsables de las operaciones examinadas. Este informe expone los comentarios, conclusiones y recomendaciones que tienen relación con los hallazgos (Contraloría General del Estado , 2003). 
También se puede indicar que el informe constituye un documento formal dirigido a la máxima autoridad, servidores y ex servidores, con el que se comunica los resultados de la evaluación realizada a la entidad.

\section{Metodología.}

La investigación se desarrolló bajo un diseño experimentalya que mediante la aplicación del examen especial se determinó las ventajas que conlleva el uso de manuales de gestión administrativa en el campo de las cuentas de depósitos a plazo fijo.

El enfoque de la presente investigación fue cuantitativo y cualitativo; la parte cuantitativa se refiere a la recolección de datos sobre la cuenta de depósitos a plazo fijo de BanEcuador, siguiendo un patrón previamente estructurado; la parte cualitativa hace relación al análisis de los resultados de la aplicación del cuestionario.

El alcance de es descriptivo y explicativo ya el estudio se basa en la necesidad de realizar un examen especial a la cuenta de depósitos a plazo fijo de BanEcuador, para luego exponer los pasos y las herramientas de auditoría que tendrían que emplearse, con el correspondiente mecanismo, al momento de la aplicación del examen, y, la finalidad fue transversal, ya que los datos relacionados a la cuenta depósitos a plazo fijo de la institución financiera BanEcuador, Sucursal Cuenca, en el período: 1 de enero al 31 de diciembre de 2018, fueron levantados y analizados en un solo momento del tiempo.

Se aplicó el método inductivo-deductivo, es decir, el método que va de lo particular a lo general, partiendo de la aplicación del cuestionario, para indagar la problemática actual de la cuenta depósitos a plazo fijo de la institución, y, en la fase posterior, para la exposición e interpretación de resultados obtenidos, lo cual permitió establecer conclusiones epecíficas del examen.

Como técnica principal, para el diagnóstico de los depósitos a plazo fijo en BanEcuador, se realizó una encuesta, mediante el instrumento del cuestionario. Para la estructuración del examen especial, se contempló cuatro puntos de vista fundamentales, referidos al financiero, gestión, control interno y cumplimiento legal. Para la parte teórica, la técnica utilizada fue la revisión, recolección, análisis y utilización de bibliografía sobre el tema.

\section{Resultados.}

- Se pudo determinar que el departamento de captaciones no mantiene un archivo adecuado, que permita verificar cada uno de los depósitos a plazo fijo que BANECUADOR realiza diariamente, se pudo evidenciar el mal manejo de los documentos pudiendo ocasionar perdidas de los mismos. 
- Se realizó una revisión de las tasas de interés sobre los depósitos a plazo fijo en BANECUADOR donde se pudo visualizar que no son competitivas con las cooperativas más relevantes de Cuenca.

- Luego de ejecutar la revisión se evidencio que falta información en la documentación que debe entregar el cliente, copias de la cedula, recibo de servicios y papeletas de votación.

- Se comprobó que no existe un control cuando se pagan los intereses.

A partir de estos resultados se considera significativo la ejecución de un Examen especial a las cuentas de depósitos a plazo fijo de la sucursal Cuenca, de Banecuador, en el periodo 01 de enero 2018 al 31 de enero 2018.

Figura 1. Esquema de la propuesta

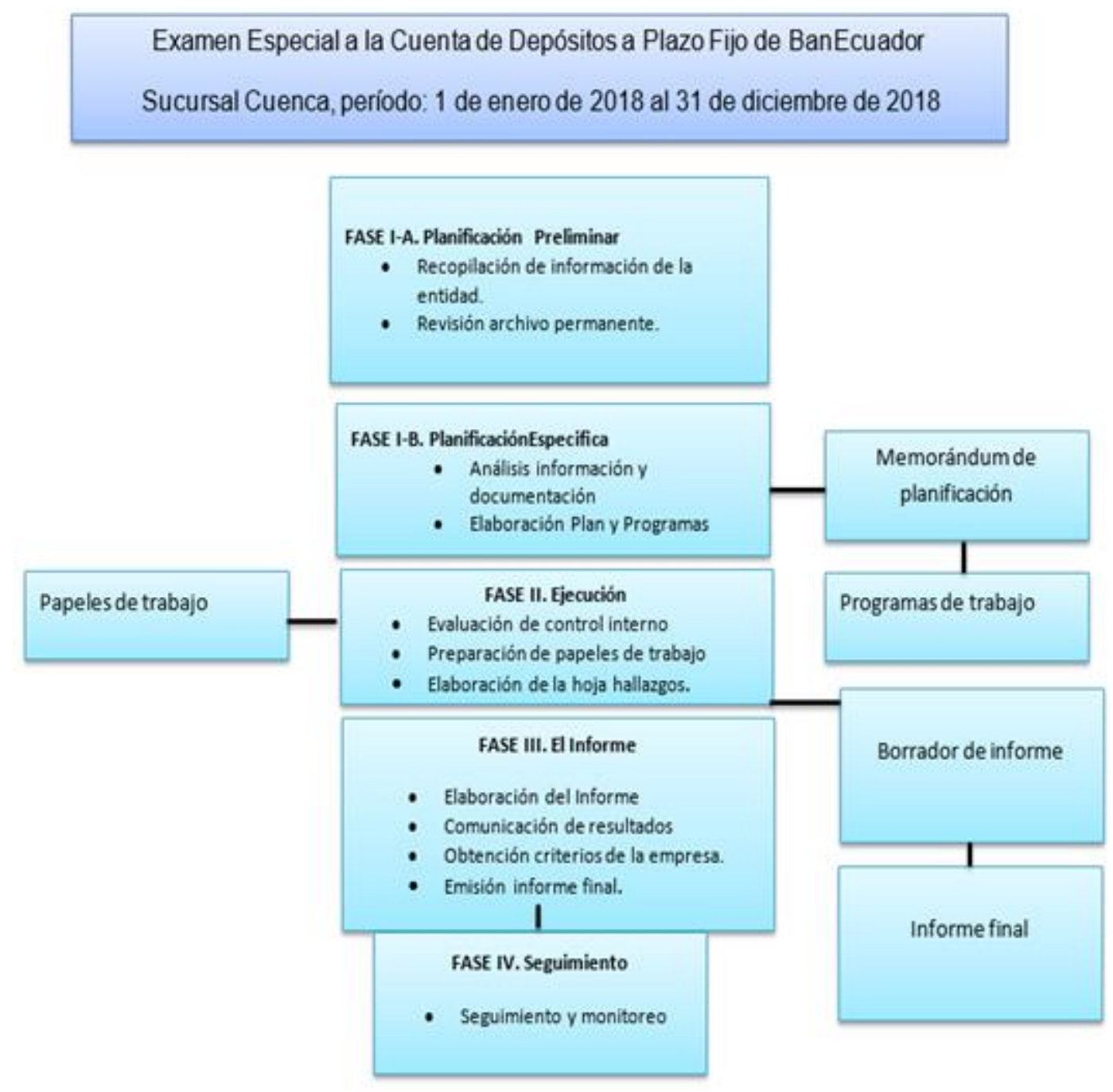

Empresa \& Gestión 


\section{Objetivo general}

Realizar un examen especial a la cuenta de DPF de BanEcuador, Sucursal Cuenca, en el período 01 de enero al 31 de diciembre de 2018, en función de que, con la realización del mismo, la entidad pueda verificar el nivel de cumplimiento de los manuales de procedimientos y normativa vigente.

\section{FASE I A.- Planificacion preliminar}

\section{$\underline{\text { Objetivos específicos de la planificación preliminar }}$}

- Establecer el enfoque del examen especial en el departamento de Captaciones.

- Verificar el nivel de cumplimiento de los procesos en la cuenta depósitos a Plazo fijo.

- Evaluar las actividades que desarrolla el departamento de Captaciones para determinar si están en relación con los objetivos planteados

Tabla 1. Cronograma planificación preliminar

\begin{tabular}{rlrcccc}
\hline N $^{\circ}$ Procedimientos & Ref/Pt & $\begin{array}{c}\text { Elaborado } \\
\text { Por: }\end{array}$ & $\begin{array}{c}\text { Revisado } \\
\text { Por: }\end{array}$ & Fecha Inicio & Fecha Fin & Obser. \\
\hline 1 & Orden de Trabajo & 2 & RLYH & RLYH & $13 / 03 / 2018$ & $13 / 03 / 2018$ \\
2 & Notificación Inicio del Examen & 3 & RLYH & RLYH & $15 / 03 / 2018$ & $17 / 03 / 2018$ \\
3 & Solicitud de Información & 4 & RLYH & RLYH & $18 / 03 / 2018$ & $19 / 03 / 2018$ \\
4 & Plan Estratégico de la Auditoria & PP001 & RLYH & RLYH & $20 / 03 / 2018$ & $22 / 03 / 2018$ \\
5 & Evaluación del C.I. General & 5 & RLYH & RLYH & $25 / 03 / 2018$ & $26 / 03 / 2018$ \\
6 & Nivel de Riesgo y Confianza & 6 & RLYH & RLYH & $27 / 03 / 2018$ & $01 / 04 / 2018$ \\
\hline
\end{tabular}

Comprobar que los programas o actividades que se realice estén sujetos a las políticas y procedimientos del banco.

Tabla 2. Cronograma recopilación información adicional.

\begin{tabular}{|c|c|c|c|c|c|c|c|}
\hline $\mathrm{N}^{\circ}$ & Procedimientos & $\mathrm{Ref} / \mathrm{Pt}$ & $\begin{array}{l}\text { Elaborado } \\
\text { Por: }\end{array}$ & $\begin{array}{l}\text { Revisado } \\
\text { Por: }\end{array}$ & $\begin{array}{l}\text { Fecha } \\
\text { Inicio }\end{array}$ & Fecha Fin & Obser. \\
\hline 1 & Visita a la empresa & CD001 & RLYH & RLYH & $21 / 03 / 2018$ & $30 / 03 / 2018$ & \\
\hline 2 & $\begin{array}{l}\text { Verificar si la información en la base legale y } \\
\text { políticas de la institución cumple en cuanto al } \\
\text { funcionamiento de la entidad. }\end{array}$ & РT003 & RLYH & RLYH & $21 / 03 / 2018$ & $30 / 03 / 2018$ & \\
\hline 3 & $\begin{array}{l}\text { Conocimiento de la misión, visión, políticas y } \\
\text { valores de la empresa. }\end{array}$ & РT004 & RLYH & RLYH & $22 / 03 / 2018$ & $03 / 04 / 2018$ & \\
\hline 2 & $\begin{array}{l}\text { Análisis de la información para seleccionar la } \\
\text { que será utilizada de acuerdo al departamento. }\end{array}$ & & RLYH & RLYH & $22 / 03 / 2018$ & $03 / 04 / 2018$ & \\
\hline 3 & $\begin{array}{l}\text { Realizar entrevistas al personal del } \\
\text { departamento involucrado. }\end{array}$ & PT005 & RLYH & RLYH & $31 / 5 / 2 / 018$ & 08/06/2018 & \\
\hline
\end{tabular}


4 Realizar la Narrativa de las entrevistas realizadas

CN001 RLYH RLYH 30/05/2018 30/05/2018

5 Identifique mediante el COSO I las áreas

críticas del Control Interno de la institución.

PT006 RLYH RLYH 01/06/2018 09/06/2018

6 Verifique la suficiencia, eficacia y

cumplimiento del Sistema de Control Interno.

PT007 RLYH RLYH 02/06/2018 $10 / 06 / 2018$

\section{FASE I B.- Planificacion especifica}

\section{$\underline{\text { Objetivos específicos de la planificación específica }}$}

- Establecer el enfoque de la auditoria gubernamental en el departamento de Captaciones.

- Verificar el nivel de cumplimiento de los procesos del departamento de Captaciones.

- Evaluar las actividades que desarrolla el departamento de Captaciones para determinar si están en relación con los objetivos planteados.

- Comprobar que los programas o actividades que se realice estén sujetos a las políticas y procedimientos del banco.

Tabla 3. Analisis de documentación e información

\begin{tabular}{|c|c|c|c|c|c|c|c|}
\hline $\mathrm{N}^{\circ}$ & Procedimientos & $\mathrm{Ref} / \mathrm{Pt}$ & $\begin{array}{l}\text { Elaborado } \\
\text { Por: }\end{array}$ & $\begin{array}{l}\text { Revisado } \\
\text { Por: }\end{array}$ & $\begin{array}{l}\text { Fecha } \\
\text { Inicio }\end{array}$ & Fecha Fin & Obser. \\
\hline 1 & $\begin{array}{l}\text { Realizar la entrevista al personal del } \\
\text { departamento involucrado. }\end{array}$ & PT02 & RLYH & RLYH & $10 / 04 / 2018$ & $10 / 04 / 2018$ & \\
\hline 2 & $\begin{array}{l}\text { Realizar el análisis del perfil professional } \\
\text { del Departamento de Captaciones. }\end{array}$ & A01 & RLYH & RLYH & $11 / 04 / 2018$ & $11 / 04 / 2018$ & \\
\hline 3 & $\begin{array}{l}\text { Analizar las políticas y procedimientos de } \\
\text { DPF. }\end{array}$ & $\mathrm{A} 02$ & RLYH & RLYH & $13 / 04 / 2018$ & $13 / 04 / 2018$ & \\
\hline 4 & $\begin{array}{l}\text { Verificar si se mantienen actualizadas las } \\
\text { políticas y procedimeintos para DPF. }\end{array}$ & $\mathrm{A} 03$ & RLYH & RLYH & $14 / 04 / 2018$ & $15 / 04 / 2018$ & \\
\hline 5 & $\begin{array}{l}\text { Verificar si las funciones establecidas } \\
\text { ayudan a cumplir las metas y objetivos } \\
\text { propuestos por el departamnto y si las } \\
\text { autoridades cumplen con sus } \\
\text { responsabilidades y labores encomendadas. }\end{array}$ & 6 & RLYH & RLYH & $14 / 04 / 2018$ & $15 / 04 / 2018$ & \\
\hline 6 & $\begin{array}{l}\text { Evalúe los procesos que se realizan en el } \\
\text { departamento de Captaciones desde la } \\
\text { creación del cliente hasta la fianlización del } \\
\text { DPF. }\end{array}$ & P07 & RLYH & RLYH & $14 / 04 / 2018$ & $15 / 04 / 2018$ & \\
\hline 7 & $\begin{array}{l}\text { Revisar los DPF para ver si cumplen con } \\
\text { las tasas establecidas por el Banco Central. }\end{array}$ & P08 & RLYH & RLYH & $14 / 04 / 2018$ & $15 / 04 / 2018$ & \\
\hline 8 & $\begin{array}{l}\text { Revisar los DPF para ver si cumplen con las } \\
\text { plazos establecidos por el Banco Central. }\end{array}$ & & RLYH & RLYH & 03/05/2019 & $03 / 05 / 2019$ & \\
\hline 9 & $\begin{array}{l}\text { Revisar los DPF para constatar si los pagos } \\
\text { de interes están como el cliente solicita. }\end{array}$ & & RLYH & RLYH & $14 / 04 / 2018$ & $15 / 04 / 2018$ & \\
\hline 10 & $\begin{array}{l}\text { Revisar DPF este con toda la } \\
\text { documentación de sustento. }\end{array}$ & P09 & RLYH & RLYH & $14 / 04 / 2018$ & $15 / 04 / 2018$ & \\
\hline
\end{tabular}


11 Revisar el archivo de los DPF para ver si están organizados cronológicamente.

$\begin{array}{ccccc}\text { P010 } & \text { RLYH } & \text { RLYH } & 14 / 04 / 2018 & 15 / 04 / 2018 \\ \text { P011 } & \text { RLYH } & \text { RLYH } & 16 / 04 / 2018 & 17 / 04 / 2018 \\ \text { PT04 } & \text { RLYH } & \text { RLYH } & 16 / 04 / 2018 & 17 / 04 / 2018 \\ \text { PT04 } & \text { RLYH } & \text { RLYH } & 16 / 04 / 2018 & 17 / 04 / 2018 \\ & & & & \\ \text { PT05 } & \text { RLYH } & \text { RLYH } & 18 / 04 / 2018 & 19 / 04 / 2018 \\ \text { PT06 } & \text { RLYH } & \text { RLYH } & 20 / 04 / 2018 & 21 / 04 / 2018 \\ \text { PT07 } & \text { RLYH } & \text { RLYH } & 22 / 04 / 2018 & 23 / 04 / 2018\end{array}$

\section{Objetivos:}

- Fundamentar, teóricamente, la importancia de los exámenes especiales y su incidencia en el cumplimiento de los manuales de procedimientos y normas vigentes relacionadas con la cuenta de depósito a plazo fijo en BanEcuador, durante el periodo 01 de enero de 2018 hasta el 31 de diciembre de 2018.

- Diagnosticar la situación operativa actual de BanEcuador, Sucursal Cuenca, de la cuenta de depósito a plazo fijo y evaluar la eficiencia y efectividad del sistema de control Interno en la cuenta depósito a plazo fijo en periodo comprendido 1 de enero de 2018 y el 31 de diciembre de 2018.

\section{Procedimientos:}

\section{Pruebas Sustantivas}

- Establecer si los montos y tasas de los de depósito a plazo fijo están bajo normativa legal vigente del Banco Central.

- Comprobar si el banco es competitivo en sus tasas de interés en el mercado financiero.

- Realizar una encuesta para ver si el personal a cargo conoce las tasas de interés del mercado financiero.

- Comprobar los los índices de liquidez del Banco deacuerdo a la Industria

- Obtener una muestra de clientes que mantienen lo de depósito a plazo fijo. 
- Constatar si existe toda la documentación para el sustento de la creación, renovación y cancelación de los de depósito a plazo fijo.

- Verificar si el archivo de la información de los de depósito a plazo fijo está siendo llevada de forma ordenada y cronológicamente.

- Detectar si existen cumplimiento de los manuales y procesos de depósito a plazo fijo.

- Revisar sie el personal de departamento de DPF cumple con el manual de procesos para el registro contable.

\section{FASE 2.- Ejecucion}

Tabla 4. Cuestionario de control interno

\begin{tabular}{|c|c|c|c|c|c|c|c|}
\hline No & Preguntas & SI & NO & N/A & PT & $\mathrm{CT}$ & Obser. \\
\hline 1 & $\begin{array}{l}\text { ¿Los plazos y tasas para aplicar en los depósitos a plazo fijo están } \\
\text { dentro del rango que establece el Banco central? }\end{array}$ & $X$ & & & 10 & 10 & \\
\hline 2 & $\begin{array}{l}\text { ¿Los funcionarios cumplen con los principios y normas establecidos } \\
\text { para registrar cada uno de los depósitos a plazo fijo? }\end{array}$ & $\mathrm{X}$ & & & 10 & 10 & \\
\hline 3 & $\begin{array}{l}\text { ¿De acuerdo al mercado financiero BanEcuador es competitivo con } \\
\text { las tasas que ofrece a sus clientes? }\end{array}$ & & $X$ & & 10 & 0 & \\
\hline 4 & $\begin{array}{l}\text { ¿La institución da a conocer los indicadores de Liquidez que para la } \\
\text { correcta toma de decisiones en la cuenta depósito a plazo fijo? }\end{array}$ & $\mathrm{X}$ & & & 10 & 10 & \\
\hline 5 & $\begin{array}{l}\text { ¿BanEcuador aplica las políticas conozca a su cliente y a su } \\
\text { empleado dentro del proceso para los depósitos a plazo fijo? }\end{array}$ & $\mathrm{X}$ & & & 10 & 10 & \\
\hline 6 & ¿Se realiza análisis a la cuenta depósito a plazo fijo en la Institución? & $\mathrm{X}$ & & & 10 & 10 & \\
\hline 7 & $\begin{array}{l}\text { ¿Existe un flujograma establecido en el banco referente a las firmas } \\
\text { de legalización en las renovaciones o captaciones nuevas? }\end{array}$ & $X$ & & & 10 & 10 & \\
\hline 8 & $\begin{array}{l}\text { ¿Se cumple los objetivos Institucionales, con las tasas depósito a } \\
\text { plazo fijo que se ofrece a los clientes? }\end{array}$ & $\mathrm{X}$ & & & 10 & 10 & \\
\hline 9 & $\begin{array}{l}\text { ¿Cada depósito a plazo fijo se encuentra con toda la documentación } \\
\text { establecida? }\end{array}$ & & $X$ & & 10 & 0 & \\
\hline 10 & $\begin{array}{l}\text { ¿La documentación de depósito a plazo fijo se encuentra archivada } \\
\text { de manera ordenada, numerada y cronológicamente? }\end{array}$ & & $X$ & & 10 & 0 & \\
\hline 11 & $\begin{array}{l}\text { ¿Los funcionarios han sido capacitados con los manuales y procesos } \\
\text { sobre depósito a plazo fijo? }\end{array}$ & $X$ & & & 10 & 10 & \\
\hline 12 & $\begin{array}{l}\text { ¿BanEcuador mantiene incentivos para los clientes que tienen } \\
\text { depósito a plazo fijo? }\end{array}$ & & $X$ & & 10 & 0 & \\
\hline \multirow[t]{2}{*}{13} & $\begin{array}{l}\text { ¿Se notifica a los clientes el vencimiento de depósito a plazo fijo } \\
\text { con anterioridad? }\end{array}$ & & $X$ & & 10 & 0 & \\
\hline & TOTAL & & & & 130 & 80 & \\
\hline
\end{tabular}




\section{VALORACIÓN}

$\mathrm{NC}=\underline{\mathrm{CT} * 100}$

$N C=80 \angle 130 * 100=61.52 \%$

Tabla 5. Determinacion del nivel de riesgo

\begin{tabular}{|c|c|c|}
\hline \multicolumn{3}{|c|}{ NIVEL DE CONFIANZA } \\
\hline Bajo & Moderado & Alto \\
\hline $15 \%-50 \%$ & $51 \%-75 \%$ & $76 \%-95 \%$ \\
\hline $85 \%-50 \%$ & $49 \%-25 \%$ & $24 \%-5 \%$ \\
\hline Alto & Moderado & Bajo \\
\hline \multicolumn{3}{|c|}{ NIVEL DE RIESGO } \\
\hline
\end{tabular}

$$
N R=100-N C
$$

$N R=100-66.67 \%=38.48 \%$

\section{CONCLUSIÓN}

Como se puede observar el nivel de confianza corresponde al $61.52 \%$ considerado alto y el nivel de riesgo $38.48 \%$ considerado moderado. Por lo tanto, este resultado indica que el enfoque para aplicar pruebas de doble propósito,en pruebas de doble propósito comprobaran tanto el cumplimiento del procedimiento de control que proporcione evidencia documentada de su realización, como la razonabilidad de la cantidad monetaria registrada en las transacciones y saldos 
FASE III .- Informe

\section{Hoja de Hallazgos}

\section{Titulo :}

Actas de entrega recepción de documentos, registros y archivos entre servidores entrantes y salientes

Condicion: (Relación cronológica de hechos. Incluir referencia de papeles de trabajo)

En la cesación y cambio de funciones de servidoras y servidores encargados de la administración, los Gerentes del Banco, no dispusieron por escrito efectuar la entrega recepción de documentos, registros y archivos a los responsables de Gerencia, Crédito y Cartera, lo que dio lugar a que los servidores encargados de la administración, suscriban las actas de entrega recepción de documentos, registros y archivos a su cargo.

Criterio: (Indicar el asunto que trata la disposición)

Los Gerentes de Sucursal, servidores del área de crédito y cartera del período examinado, incumplieron el artículo 77, numeral 1, letra a); numeral 3, letras b), c) y d) de la Ley Orgánica de la Contraloría General del Estado, e inobservaron el artículo 110 "Entrega de bienes y archivos", del Reglamento General a la Ley Orgánica del Servicio Público que dispone:

“...En los casos de cesación de funciones, salvo por muerte la o el servidor, se deberá suscribir obligatoriamente un acta de entrega recepción de los bienes y archivos bajo su responsabilidad...".

Causa(s): (Acciones no realizadas por los responsables y que ocasionaron la desviación)

La omisión de las servidoras y servidores públicos en la entrega recepción de documentos, registros y archivos mediante actas, en las áreas de Gerencia de Sucursal, Crédito y Cartera.

\section{$\underline{\text { Efecto }}$}

Ocasionó que la entidad no mantenga un archivo completo de la documentación que sirva como sustento de su gestión, toma de decisiones y control posterior. 


\section{Conclusiones}

Los servidores salientes y entrantes no formularon actas de entrega recepción de documentos, registros y archivos, situación que no permitió contar con información suficiente y oportuna para la toma de decisiones y control posterior.

$\underline{\text { Recomendaciones (Procedimientos sugeridos para eliminar causas establecidas) }}$

\section{A la Gerente de Sucursal}

1. Dispondrá a los servidores y servidoras de la entidad, que cuando sean remplazados temporal y definitivamente de la función asignada, realicen la entrega recepción de bienes, archivos y registros a su cargo, con el servidor entrante; de dicho procedimiento, se dejará constancia escrita en un acta en la que deberán constar las novedades detectadas.

\section{Conclusiones.}

- En la cesación y cambio de funciones de servidoras y servidores encargados de la administración, los Gerentes del Banco, no dispusieron por escrito efectuar la entrega recepción de documentos, registros y archivos a los responsables de Gerencia, Crédito y Cartera, lo que dio lugar a que los servidores encargados de la administración, suscriban las actas de entrega recepción de documentos, registros y archivos a su cargo.

- La aplicación de un Examen Especial técnico y profesional, permitió conocer la aplicación de los manuales en la cuenta de Depósitos a Plazo Fijo, establecer posibles errores, obtener las diferentes observaciones y recomendaciones, lo que contribuye al fortalecimiento de la gestión financiera y suscitar su eficiencia operativa.

- Otro de los hallazgos que se pudo determinar fue el hecho de que el examen especial a la cuenta de depósitos a plazo fijo sí permite la comprensión de las falencias y fortalezas de una entidad bancaria, por lo cual se llega a la conclusión de que BanEcuador sí puede llegar a ser competitivo dentro del mercado financiero, ello por medio del desarrollo de un control interno, dando cabida a una organización de carácter sistémico, en donde la comunicación fluya de forma asertiva entre todos los departamentos que son parte de la institución financiera.

- Se propone mantener un registro correcto de los depósitos a plazo fijo tanto en la tasa, tiempos, segundo beneficiario y pagos de interés con la finalidad de control interno. 
Igualmente debe mantenerse un archivo ordenado en forma cronológica de la documentación fuente; lo que permitirá su fácil acceso, localización e identificación oportuna de cada uno de los depósitos a plazo fijo. Por último se sugiere una reunión con las altas gerencias de la matriz en donde se pueda tomar en cuenta las tasas de interés que ofrece el banco llegando a mantener una política de negociación para los clientes en caso de montos altos para cambio de tasas de interés

\section{Referencias Bibliográficas}

Aldana, S., Vereda, F., Hidalgo-Alvarez, R., \& de Vicente, J. (2016). Facile synthesis of magnetic agarose microfibers by directed selfassembly. Polymer, 93, 61-64.

Alvarez, M. (1996). Manual para elaborar manuales de políticas y procedimientos. México: Panorama editorial.

Álvarez Torres, Martin G (1996) Manual para elaborar manuales de políticas y procedimientos. México: Panorama.

Arens, A. (1996). Auditoría, un Enfoque Integral (Primera ed.). México D.F.: Prentice Hall Hispanoamericana.

BanEcuador. (2016). Depósito a plazo fijo. Obtenido de Depósito a plazo fijo: https://www.banecuador.fin.ec/a-quien-financiara/inversiones/

Bayas, S., Astudillo, N., Aulestia, P., Calderón, P., Cacarín, H., \& Cepeda, M. (Marzo de 2012). Fuentes de financiamiento e inversión a corto plazo. Obtenido de Fuentes de financiamiento e inversión a corto plazo: http://portal.uasb.edu.ec/UserFiles/381/File/Fuentes_de_Financiamiento\%20PRESENTA CION.pdf

Bayas, S., Marco, C., \& Astudillo, N. (2012). Fuentes de Financiamiento e Inversión a Corto Plazo. Quito: Universidad Andina Simón Bolívar.

Bhat, S., Tripathi, A., \& Kumar, A. (2010). Supermacroprous chitosan-agarose-gelatin cryogels. in vitro characterization and in vivo assesment for cartilage tissue engineering. Journal of the Royal Society Interface, 1-15. 
Bossis, G., Marins, J., Kuzhir, P., Volkova, O., \& Zubarev, A. (2015). Functionalized microfibers for field-responsive materials and biological applications. Journal of Intelligent Material Systems and Structures, 1-9.

Bové, J. M. (2012). Repercuciones empresariales de la evalución del control interno realizada por auditores independientes. Revista Española de financiamiento y contabilidad, 107124.

Campos, R. (2015). Fundamentos de la administración financiera. México : Red Tercer Milenio.

Cañibano, L. (1996). Curso de auditoría contable. Madrid: Pirámide.

Contraloría General del Estado . (2003). Ecuador .

Contraloría General del Estado. (2015). Ley Orgánica de la Contraloría General del Estado. Quito: Congreso Nacional.

Cortés, J., Puig, J., Morales , J., \& Mendizábal, E. (2011). Hidrogeles nanoestructurados termosensibles sintetizados mediante polimerización en microemulsión inversa. Revista Mexicana de Ingeniería Química., 10(3), 513-520.

Cuéllar, A. (2003). Teoría general de la auditoría y revisoria fiscal. Colombia : Editorial Patria.

Defliese, P., Jaenicke, H., Gnospelius, R., \& Sullivan, J. (1996). Auditoría Montgomery (Primera ed.). México D.F.: Limusa.

Dias, A., Hussain, A., Marcos, A., \& Roque, A. (2011). A biotechnological perspective on the application of iron oxide magnetic colloids modified with polysaccharides. Biotechnology Advances 29, 29, 142-155.

Estrada Guerrero, R., Lemus Torres, D., Mendoza Anaya, D., \& Rodriguez Lugo, V. (2010). Hidrogeles poliméricos potencialmente aplicables en Agricultura. Revista Iberoamericana de Polímeros, 12(2), 76-87.

Franklin, E. B. (2007). Auditoría administrativa. México: Pearson Educación .

García-Cerda, L., Rodríguez-Fernández, O., Betancourt-Galindo, R., Saldívar-Guerrero, R., \& Torres-Torres, M. (2003). Síntesis y propiedades de ferrofluidos de magnetita. Superficies y Vacío., 16(1), 28-31.

Ilg, P. (2013). Stimuli-responsive hydrogels cross-linked by magnetic nanoparticles. Soft Matter, 9, 3465-3468. 
Lewitus, D., Branch, J., Smith, K., Callegari, G., Kohn, J., \& Neimark, A. (2011). Biohybrid carbon nanotube/agarose fibers for neural tissue engineering. Advanced Functional Materials, 21, 2624-2632.

Ley Orgánica de la Contraloría General del Estado. (2015). Del sistema de control intero . Ecuador.

Lin, Y.-S., Huang, K.-S., Yang, C.-H., Wang, C.-Y., Yang, Y.-S., Hsu, H.-C., . . Tsai, C.-W. (2012). Microfluidic synthesis of microfibers for magnetic-responsive controlled drug release and cell culture. PLOS ONE, 7(3), 1-8.

Luna, Y. B. (2012). Auditoría Integral: Normas y procedimientos. Colombia: Ecoe Ediciones .

Mantilla, O., Herrera, L., \& Pinilla. (2005). El deber ser de la auditoría. Colombia : Universidad Icesi .

Manual de Auditoría Financiera Gubernamental. (2001). Generalidades . Ecuador .

Mejía, E., Montilla, O., \& Montes, y. (2005). Contabilidad y racionalismo crítico. Colombia : Universidad Libre .

Montilla Galvis, O. d., \& Herrera Marchena, L. G. (2006). El deber ser de la auditoría. Calí: Universidad ICESI.

Moore Stephens. (2019, 06 01). moorestephens. Retrieved from moorestephens: http://ec.moorestephens.com/

Plan Nacional de Desarrollo 2017-2021. (2017). Más sociedad, mejor Estado. Ecuador : Secretaría Nacional de Planificación y Desarrollo.

Ruiz Estrada, G. (2004). Desarrollo de un Sistema de liberación de fármacos basado en nanopartículas magnéticas recubiertas con Polietilénglicol para el tratamiento de diferentes enfermedades. Madrid: Universidad Autónoma de Madrid. Departamento de Física Aplicada.

Sandoval, M. H. (2012). Introduccion a la Auditoría. México: Red Tercer Milenio.

Santillana, G. J. (2004). Establecimiento del Sistema de Control Interno. México D.F.: International Thomson Editores, S. A. de C. V.

Song, J., King, S., Yoon, S., Cho, D., \& Jeong, Y. (2014). Enhanced spinnability of narbon nanotube fibers by surfactant addition. Fiberes and Polymers, 15(4), 762-766. 
Sotomayor, A. (2008). Auditoría administrativa : Proceso y aplicación. México: Mc Graw Hill.

Tartaj, P., Morales, M., González-Carreño, T., Veintemillas-Verdaguer, S., \& Serna, C. (2005). Advances in magnetic nanoparticles for biotechnology applications. Journal of Magnetism and Magnetic Materials, 290, 28-34.

Wulff-Pérez , M., Martín-Rodriguez, A., Gálvez-Ruiz, M., \& de Vicente, J. ( 2013 ). The effect of polymer surfactant on the rheological properties of nanoemulsions. Colloid and Polymer Science, 291, 709-716.

Zamora Mora, V., Soares, P., Echeverria, C., Hernández , R., \& Mijangos, C. (2015). Composite chitosan/Agarose ferrogels for potential applications in magnetic hyperethermia. Gels., 1 , 69-80. 


\section{PARA CITAR EL ARTICULO}

Yuquilima Heras, R., Narváez Zurita, C., Torres Palacios, M., \& Ordoñez Parra, Y. (2019). Examen especial a la cuenta de depósitos a plazo fijo de BanEcuador sucursal Cuenca, por el período comprendido entre el 01 de enero al 31 de diciembre de 2018. Visionario Digital, 3(2.1.), 527-549. https://doi.org/10.33262/visionariodigital.v3i2.1.587

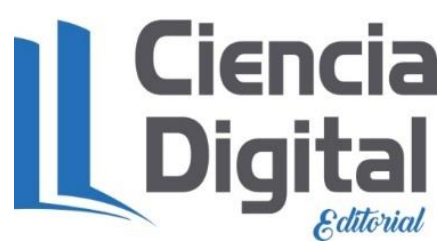

El artículo que se publica es de exclusiva responsabilidad de los autores y no necesariamente reflejan el pensamiento de la Revista Ciencia Digital.

El artículo queda en propiedad de la revista y, por tanto, su publicación parcial y/o total en otro medio tiene que ser autorizado por el director de la Revista Ciencia Digital.
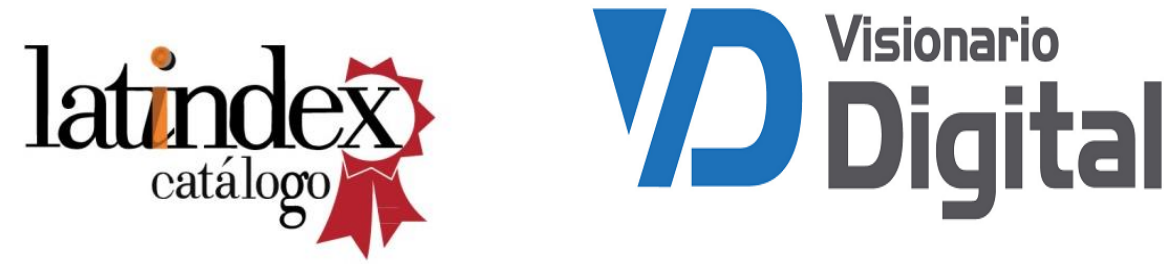